(C) 2017 IEEE. Personal use of this material is permitted. Permission from IEEE must be obtained for all other uses, in any current or future media, including reprinting/republishing this material for advertising or promotional purposes, creating new collective works, for resale or redistribution to servers or lists, or reuse of any copyrighted component of this work in other works. 


\title{
Low-Profile, Electrically Small, Huygens Source Antenna with Pattern-Reconfigurability that Covers the Entire Azimuthal Plane
}

\author{
Ming-Chun Tang, Senior Member, IEEE, Boya Zhou, Student Member, IEEE, and Richard W. \\ Ziolkowski, Fellow, IEEE
}

\begin{abstract}
A pattern-reconfigurable, low-profile, efficient, electrically small, near-field resonant parasitic (NFRP), Huygens source antenna is presented. The design incorporates both electric and magnetic NFRP elements. The electric ones are made reconfigurable by the inclusion of a set of p-i-n diodes. By arranging these electric and magnetic NFRP elements properly, a set of three Huygens sources are attained, each covering a $120^{\circ}$ sector. Pattern reconfigurability is obtained by switching the diodes on or off; it encompasses the entire $360^{\circ}$ azimuth range. A prototype was fabricated and tested. The numerical and experimental studies are in good agreement. The experimental results indicate that in each of its instantaneous states at $\mathrm{f}_{\mathbf{0}}=\mathbf{1 . 5 6 4}$ GHz, the antenna provides uniform peak realized gains, front-to-back ratios, and radiation efficiencies, respectively, as high as $3.55 \mathrm{dBi}, 17.5 \mathrm{~dB}$, and $84.9 \%$, even though it is electrically small: $k a=0.92$, and low profile: $0.05 \lambda_{0}$.
\end{abstract}

Index Terms - Electrically small antennas, Huygens source antennas, low profile antennas, near-field resonant parasitic elements, pattern-reconfigurable antennas

\section{INTRODUCTION}

$\mathrm{T}_{\mathrm{de}}^{\mathrm{he}}$ e accelerated demands on wireless technologies have driven hardware to be more compact, integrated, portable, and lower cost while nevertheless promising better, smarter and more multifunctional performance characteristics. They have spawned the development of a plethora of high-performance electrically small antennas (ESAs) to satisfy the requirements in

Manuscript received on Aug. 05, 2016; revised on Nov. 30, 2016; and accepted on Dec. 18, 2016

This work was supported in part by the National Natural Science Foundation of China contract number 61471072, in part by Graduate Scientific Research and Innovation Foundation of Chongqing, China contract number CYB16047, in part by Chongqing Postdoctoral Special Funding Project contract number xm2016022, in part by China Postdoctoral Science Foundation contract number 2016M590860, in part by Opening subject of State Key Laboratory of Millimeter Waves contract number K201732, and in part by the Australian Research Council grant number DP160102219.

M.-C. Tang is with the College of Communication Engineering, Chongqing University, Chongqing, 400044, China and with the State Key Laboratory of Millimeter Waves, Nanjing, 210096, China (E-mail: tangmingchun@cqu.edu.cn).

B. Zhou is with the College of Communication Engineering, Chongqing University, Chongqing, 400044, China.

R. W. Ziolkowski is with the University of Technology Sydney, Global Big Data Technologies Centre, Ultimo NSW 2007, Australia and with The University of Arizona, Department of Electrical and Computer Engineering, Tucson, AZ 85721 USA (E-mail: ziolkowski@ece.arizona.edu). different scenarios. These advances have included multi-segment wire antennas [1]; anisotropic metamaterial-based antennas [2]; composite right/left-handed transmission line based antennas [3]; capacitively coupled, folded monopole antennas [4]; conical folded dipole antennas [5]; metal-mountable radio-frequency-identification (RFID) tag antennas [6]; folded helix/planar meander-line antennas [7]; self-excited electromagnetic bandgap (EBG) resonator antennas [8]; and near-field resonant parasitic (NFRP) antennas [9]. Unfortunately, because their radiation characteristics are not reconfigurable, many of these ESAs cannot individually meet system requirements that cope with changing scenarios.

An effective, low-cost, and space-saving solution to these issues has been reconfigurable technologies. Reconfigurability empowers many desirable properties such as tunable operating frequencies, radiation patterns, and polarization states. It is an excellent alternative to the common solution, i.e., utilizing several fixed antennas to meet the requirements $[10,11]$. In particular, pattern-reconfigurable antennas have many known admirable capabilities, including dynamic beam-switching, direction-finding, null scanning, and incoming interferenceavoiding. They have been extensively investigated for civil and military applications $[12,13]$. While there have been many types of pattern-reconfigurable antennas developed in recent years, e.g., dielectric resonator antennas [14], graphene-metal loop antennas [15], Yagi-Uda antennas [16-18], microstrip stacked array antennas [19], and implantable antennas [20], there have been very few electrically small designs reported.

Because of their electrical size, i.e., $k a<1$ (where $a$ is the radius of the smallest sphere that completely encloses the antenna system at the operational frequency, $f_{0}$, and $k=2 \pi / \lambda_{0}=$ $2 \pi f_{0} / \mathrm{c}$ is the corresponding free space wave number), ESAs generally have no directional performance of note. They simply are constrained physically to act as electric or magnetic dipole radiators. Thus, their directivity is deemed to be too low for many applications $[21,22]$. Nevertheless, several efforts have been devoted to achieve pattern-reconfigurable ESAs. These include Yagi-Uda monopole ESAs [23] and coplanar waveguide (CPW) -fed ESAs [24]. The former is based on generating two oppositely directed beams by switching diodes on the bottom of the directors. The result was reconfigurable, but the 3-dB beamwidth coverage was quite limited [23]. In a previous study [24], we accomplished pattern-reconfigurability by changing the gap position in a ground-connected 
capacitively loaded semi-loop near-field resonant parasitic (NFRP) element of a uniplanar ESA. Unfortunately, this design did not maintain the same frequency of operation as the pattern was reconfigured because the changes in the gap positions produced different effective electric lengths of the semi-loop. Nevertheless, if it were possible, it would be highly desirable to achieve a pattern-reconfigurable ESA design that is both efficient and directional at a fixed operational frequency with a wide-angle coverage to meet various complicated and changing scenarios from a limited-size wireless platform.

Huygens source antennas possess the admirable advantage of having significant directional radiation performance without having to incorporate director/reflector elements [25], periodic electromagnetic band-gap (EBG) structures [26, 27], metallic cavities [28], or relatively large ground planes. They have been extensively investigated over the past several years [e.g., 29-32]. We have recently achieved several electrically small Huygens source antennas, e.g., planar, end-fire radiating [33]; ultra-low profile, broadside radiating [34, 35]; and non-Foster [36] versions.

In this paper, we introduce a pattern-reconfigurable, low-profile, efficient, electrically small Huygens source antenna that covers the entire azimuth plane, which is quite different from our previously reported passive [33-35] and active [36] versions. The antenna design is demonstrated in Section II. To understand the design principles, the physical mechanisms of its reconfigurable operation are discussed in a comprehensive manner in Section III. Its simulated reconfigurable performance characteristics are presented in Section IV. A prototype antenna was fabricated and experiments were performed to validate those results. The experiments and their outcomes are also described in Section IV. In contrast to the extensive set of electrically large reconfigurable systems mentioned previously [12-20], the measured results confirm the efficacy of this electrically small, reconfigurable antenna.

The experimental results are compared to their simulated values; they are in good agreement. In particular, they verify that the prototype exhibits reconfigurability that covers the entire azimuth plane at a fixed frequency point, $1.564 \mathrm{GHz}$, with only three states. Moreover, it is found to have design and performance characteristics that are superior to previously reported Yagi - Uda [23] and CPW-fed [24] ESAs. The predicted nearly complete impedance match, reasonably high radiation efficiency, and stable and uniform directional radiation patterns for all three states were confirmed. Finally, some conclusions are drawn in Section V.

We note that all of the metallic elements modeled in the antenna designs were chosen to be copper with its known material parameters: $\varepsilon_{r}=1.0, \mu_{r}=0.999991$ and bulk conductivity $\sigma=5.8 \times 10^{7} \mathrm{Siemens} / \mathrm{m}$. The design and the fabricated prototype employed Rogers Duroid ${ }^{\mathrm{TM}} 5880$ laminate sheets whose relative permittivity $\varepsilon_{r}=2.2$, relative permeability $\mu_{r}=1$, and loss tangent $\tan \delta=0.0009$; whose thickness was $0.787 \mathrm{~mm}$ (31 mils); and whose copper cladding thickness was $0.5 \mathrm{oz}(0.017 \mathrm{~mm})$. All of the numerical simulations and their optimizations were performed using the frequency domain, finite element-based ANSYS/ANSOFT High Frequency Structure Simulator (HFSS), version 13.0 [37].

\section{ReConfigurable Huygens Source ANTENNA DESIGN}

The reconfigurable Huygens source antenna is illustrated in Fig. 1. Its optimized structural parameters are listed in Table I. The operating principles were derived from the design in [33, 34], i.e., electric and magnetic NFRP elements are combined with a driven electric antenna to achieve the desired Huygens source behavior.

As is shown in Figs. 1(a) and 1(b), the proposed antenna is a z-oriented stack of two parallel Rogers Duroid ${ }^{\mathrm{TM}} 5880$ laminate disks. The bottom disk has a slot-based electric NFRP element etched out of its upper side. A coax-fed electric tripole antenna is printed on the bottom side of the second, top disk; the coax passes completely through the bottom disk. A set of reconfigurable magnetic NFRP elements are etched on the top side of the top disk. Each consists of a $120^{\circ}$ sectoral capacitively loaded loop (CLL). The slotted regions on the bottom disk are matched to those sectors; they are interconnected in series with three p-i-n (PIN) diodes.

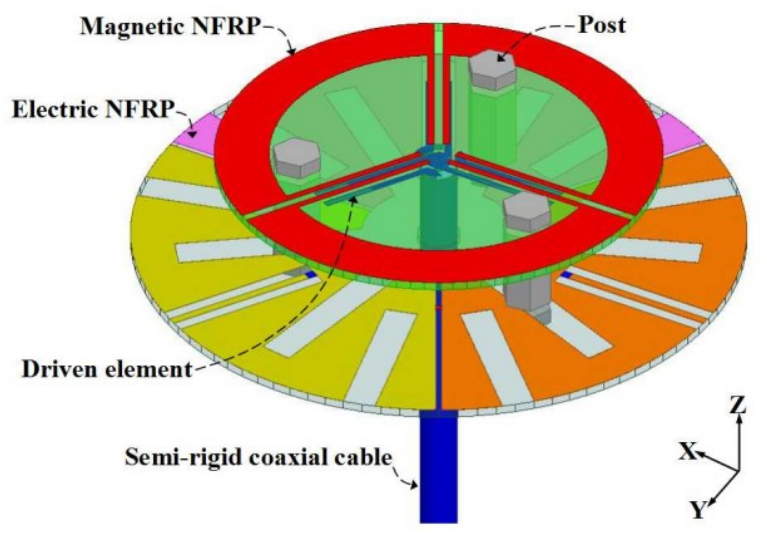

(a)

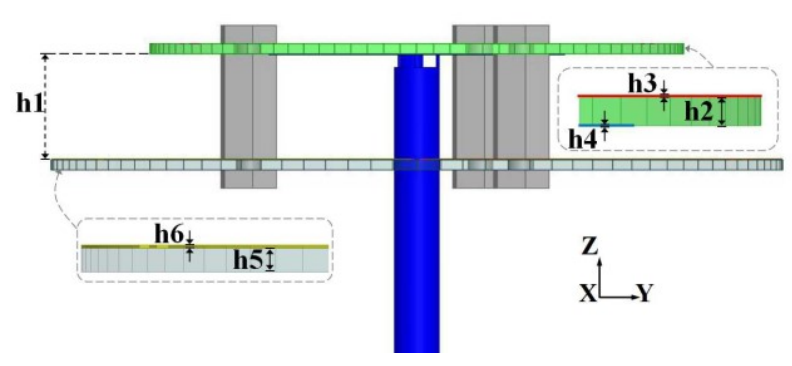

(b) 


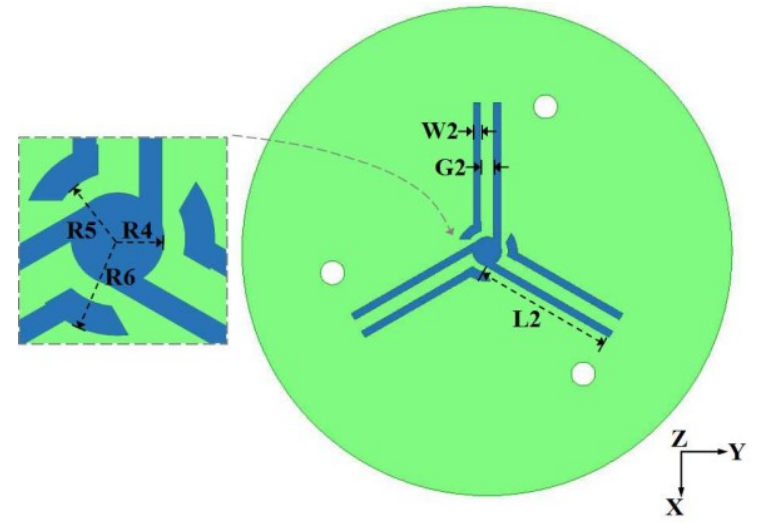

(c)

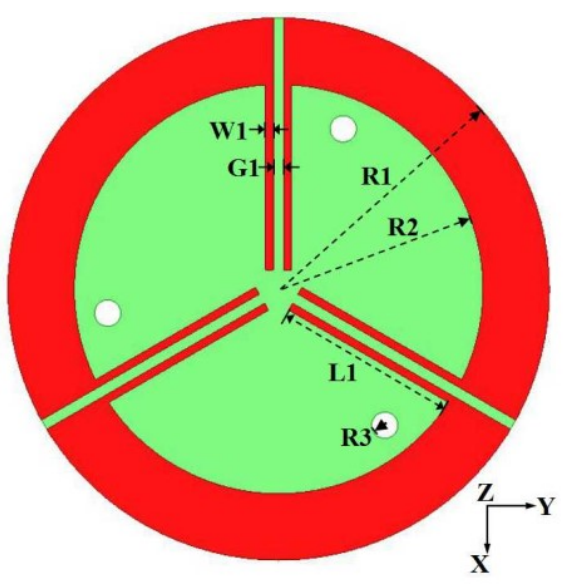

(d)

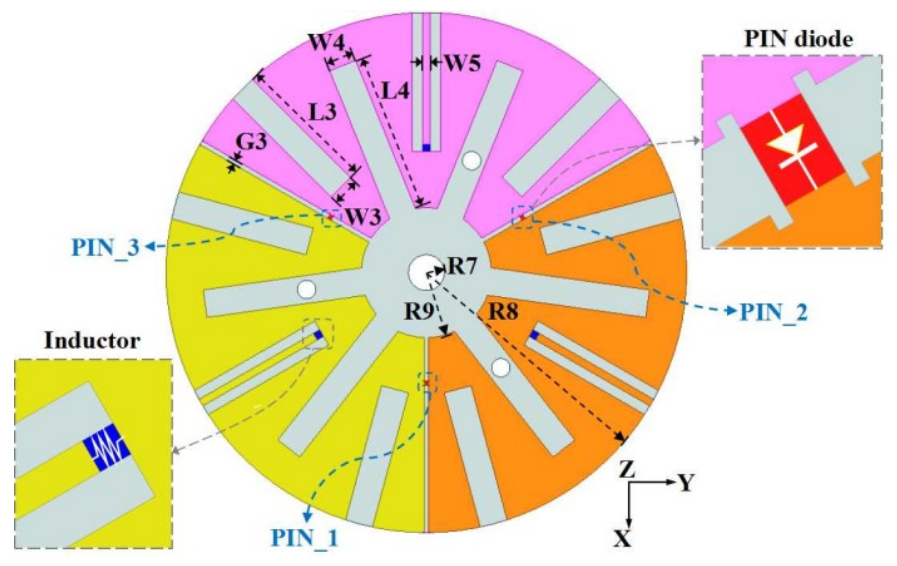

(e)

Fig. 1 The pattern-reconfigurable, low profile, electrically small, Huygens source antenna design. (a) 3-dimensional isometric view, (b) side view, (c) top view of the driven element, (d) top view of the magnetic NFRP elements, and (e) top view of the reconfigurable electric NFRP elements.

The driven tripole electric antenna is shown in Fig. 1(c). It is specifically designed to accommodate the 120-degree centrally symmetric structure of the magnetic NFRP element. The center conductor of the $50 \Omega$ coax-feed line is directly connected to an inner circular patch that has three straight strip elements radiating outwards from it to form a Y-shaped structure. The resulting Y-shaped structure also has three parasitic strips associated with each of its arms. These three pairs of rectangular strips were designed to provide a homogeneous capacitive coupling level between them and the corresponding three magnetic NFRP CLL elements. For one typical pair of strips, the one on the right is the extension from the central circular patch. The second strip of a pair is the parasitic; it is directly connected to the outer wall of the coax cable through the arc-shaped tab on its inner end.

TABle I. The Optimized Design Parameters of the ReConfigurable Huygens Source ANTENNA (All Dimensions ARe in Millimeters)

\begin{tabular}{|l|l|l|l|l|}
\hline$h 1=8$ & $h 2=0.787$ & $h 3=0.017$ & $h 4=0.017$ & $h 5=0.787$ \\
\hline$h 6=0.017$ & $L 1=13.92$ & $L 2=12.06$ & $L 3=14.96$ & $L 4=17.20$ \\
\hline$W 1=0.59$ & $W 2=0.6$ & $W 3=3$ & $W 4=3$ & $W 5=0.8$ \\
\hline$R 1=20.4$ & $R 2=15.4$ & $R 3=1$ & $R 4=1.2$ & $R 5=1.56$ \\
\hline$R 6=2.5$ & $R 7=1.94$ & $R 8=28$ & $R 9=7$ & $G 1=0.8$ \\
\hline$G 2=1.1$ & $G 3=0.3$ & \multicolumn{4}{|c|}{ Null } \\
\hline
\end{tabular}

To enable the mechanical stability of the antenna system during its installation and all of the experiments, a KTG 141-50 type, semi-rigid, $50 \Omega$ coaxial cable from Shenzhen Kansai Industrial LTD was selected to feed it. As shown in Figs. 1(a) and 1(b), it goes through a circular hole in the center of the disk. Its center conductor is soldered directly to the driven element at the bottom of the upper substrate. Also as shown, three hexagonal plastic nuts and screws were uniformly installed to support the two substrates and to assure that the two substrates are parallel to each other. The distance between the two disks is $8 \mathrm{~mm}$ and both of them are oriented perpendicular to the coaxial cable.

The magnetic NFRP element lies on the upper layer of the top disk as shown in Fig. 1(d). Three identical, printed $120^{\circ}$ sectoral CLLs are oriented and positioned to match with the tripole legs. The two stubs at the end of each CLL are perpendicular to each other to form a well-defined capacitive gap. The loop currents generated in these elements supply the desired magnetic dipole response.

By adjusting the distance between the left and right strips (G2) of the tripole and by altering their widths (W2), excellent impedance match to the $50 \Omega$ source was easily achieved in our simulation studies. There was no need for any complicated or expensive feed network. This behavior is similar to that found with our other reported NFRP ESAs [9]. However, the shapes of the magnetic and driven elements of this design are quite different, most notably from our reported NFRP magnetic ESAs in [9, 33-36]. Nonetheless, the basic physics is the same. The reason for the physical differences will be illustrated in detail in Section III (a).

The top surface of the lower disk is shown in Fig. 1(e). It is separated into $3120^{\circ}$ sectors. The copper regions are displayed in three different colors; the slots are shown in grey. All of the 
thick slots are centered on the center of the disk. There are three additional pairs of straight rectangular slots that reside below the legs of the driven tripole element. As shown in the sub-plot of Fig. 1(e), a $56 \mathrm{nH}$ coil inductor is installed at each inner end of the conducting strip formed by the slot pairs to block the RF signal from entering into the DC bias network. Each of the outer ends of these strips is used as the connection point for the DC feeder lines required to control the states of the diodes. The gaps between the sectors are equal (G3).

Three M/A-COM MA4GP907 PIN diodes are incorporated into this electric NFRP element, one across each of the three sector gaps. Each diode thus connects two sectors. In order to ensure their positions were the same distance away from the center point of the disk when they were installed, two pairs of small rectangular slots, which serve as were etched on both sides of the gap, all four slots being $0.1 \mathrm{~mm} \times 0.06 \mathrm{~mm}$ in size. These slots provided two pads, $0.385 \mathrm{~mm} \times 0.06 \mathrm{~mm}$ in size on each side of the gap, to act as markers for the placement of each diode. This arrangement is shown in the sub-plot of Fig. 1(e). We note that the presence of the small-sized four slots and two pads have little influence on the antenna performance. Moreover, these features were created with a chemical etching method whose practical tolerances are within $\pm 0.02 \mathrm{~mm}$.

As defined by its datasheet, each diode was modeled in a simulation as a $5.2 \Omega$ resistor for the $\mathrm{ON}$ state and as a $0.025 \mathrm{pF}$ capacitor for the OFF state [38]. For an easy description of their on and off states, we have denoted these diodes as PIN_1, PIN_2 and PIN_3 in terms of their three different positions shown in Fig. 1(e). Then, for instance, when PIN_1 is ON, while PIN_2 and PIN_3 are OFF, the bottom disc can be treated simply as two NFRP electric elements that are both symmetric with respect to the $x$-axis. This will be denoted as State A. Likewise, State B will denote PIN_2 is ON and PIN_3 and PIN_1 are OFF. State C will denote PIN_3 is ON and PIN_1 and PIN_2 are OFF. Moreover, one has an electrical length along the $\mathrm{y}$-axis that is twice as long as the other one. For instance, in State A, this length difference causes a phase response between the two electric NFRP elements that produces a radiation sum pattern along the $+x$-axis (i.e., along the end-fire direction). Furthermore, the combination of the magnetic dipole pattern from the upper disk element and the electric dipole sum pattern from the lower disk elements provide the desired Huygens source behavior. This physics will be explained next.

\section{DESIGN PRINCIPLES}

In order to clarify the physical principles of operation of the pattern-reconfigurable Huygens antenna, we have performed a comprehensive investigation of its behavior. We summarize the understanding gleamed from these studies in this section. They included examining the magnetic ESA alone, the phase responses of the reconfigurable electric NFRP element, and the $E$-field distribution in the near field of the antenna system.

\section{A. Behavior of the set of magnetic NFRP elements}

As one of the two main radiators in the reconfigurable ESA, the magnetic NFRP element plays an important role in forming its directional radiation pattern [33-36]. To confirm its operation, a series of ESAs formed with only a coax-fed multi-arm driven element and a corresponding number of magnetic NFRP CLL elements was investigated. The geometry of this series along with the resulting current distributions on the various components of the antenna and the resulting directivity patterns are compared in Fig. 2(a).

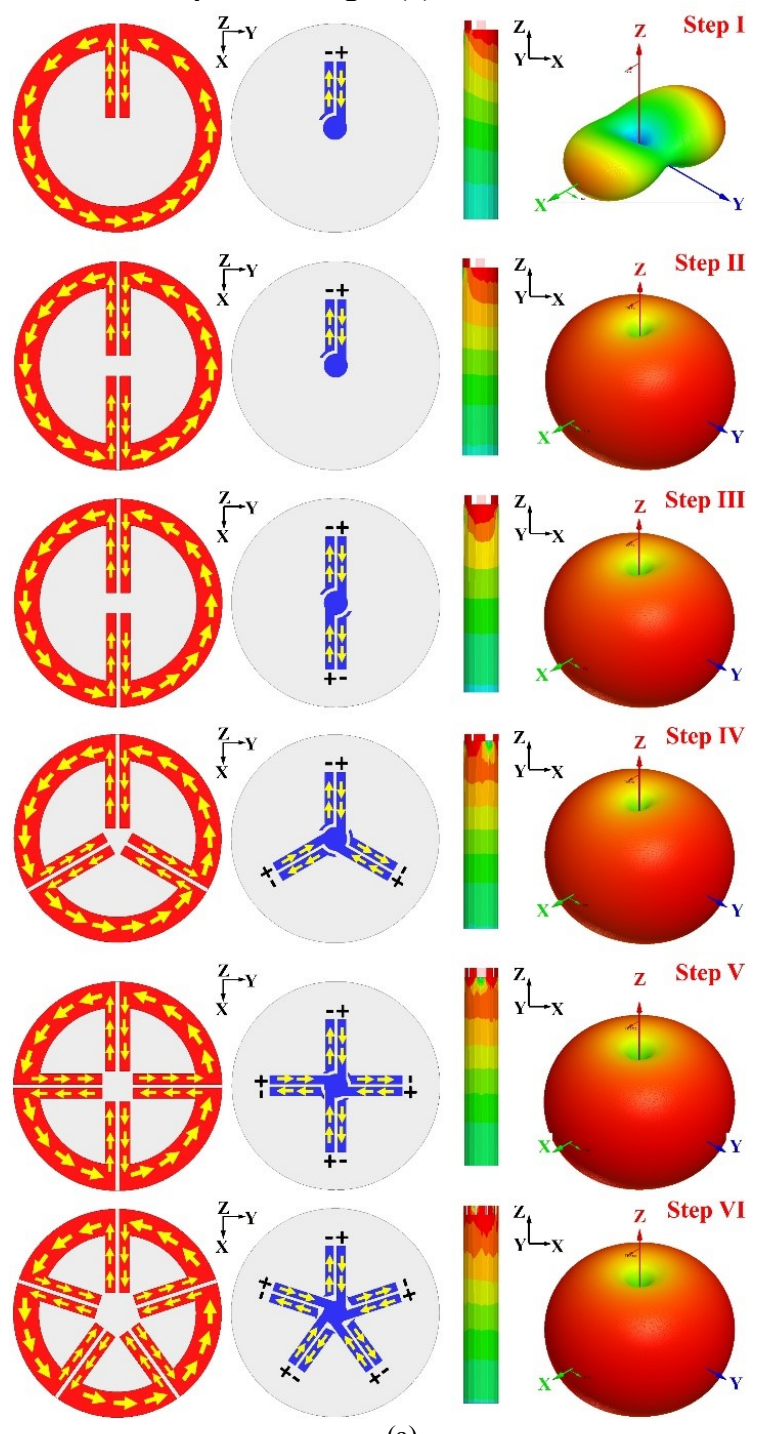

(a)

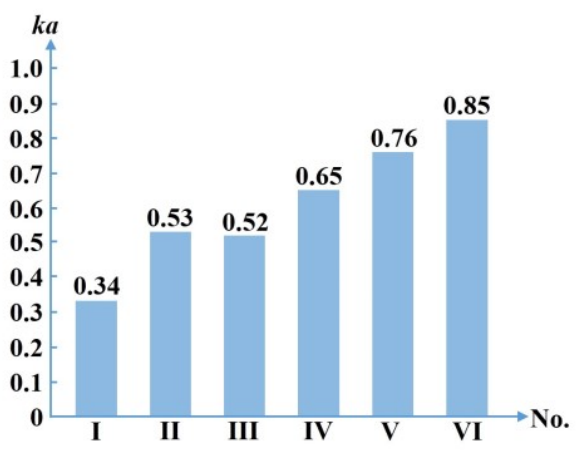

(b)

Fig. 2 Evolution of the behaviors of six magnetic NFRP ESAs, Steps I-VI. (a) The simulated current distribution behaviors on the upper surface of the NFRP CLL elements, on the upper surface of the driven elements, and on the outer surface of the coax feedline, and the corresponding 3D directivity patterns of each of the antennas in the series; and (b) a summary of their electrical sizes. 
The evolution of the behavior of these magnetic dipole ESAs is displayed as the number of their NFRP CLL elements and/or the number of arms in the driven dipole antenna increases. As expected, a loop current mode is induced on each of the NFRP elements. This produces the desired magnetic dipole mode of operation [21]. A summary of the corresponding electrical sizes of these ESAs is given graphically in Fig. 2(b). The $k a$ value increases as more CLL elements are introduced. The resonance frequency of a CLL element is mainly controlled by its length (i.e., its effective inductance) and the size of its gap (i.e., effective capacitance). Because, for instance, a quarter sector version is smaller in length than a half one for a given radius, the overall radius of the disk has to be increased to increase the sector sizes to recover the desired resonance frequency with each element.

For the first antenna in the series, Step I, there is only a single CLL and there are two corresponding strips in the driven element. Because the NFRP element is not symmetric with respect to the z-axis, an inhomogeneous current distribution results on it. It prevents this ESA from exhibiting a smooth omni-directional radiation pattern behavior in the xy plane. Thus, Step II has two CLLs placed above the same driven element to eliminate this asymmetry. It is clearly observed that it achieves a very much improved omni-directional pattern. This particular combination of CLL and driven elements was used successfully in earlier passive Huygens source ESA designs [33, 34].

Concomitantly, it should be noticed that in both of these first two designs the transition from the driven strips to the coaxial feedline is not completely balanced. Consequently, there is some stray inhomogeneous current distributed on the outer wall of the coax cable; it cannot be neglected. It creates some electric dipole mode along the $\mathrm{z}$-axis which, in turn, tilts the pattern some. More importantly, it would also mean that an asymmetry would be present in the radiation performance over the three sectors and, hence, it would impact the reconfigurability. This phenomenon has been confirmed in our simulation study. Therefore, it is necessary to make the reconfigurable pattern in every state as uniform as possible, i.e., equal impedance matching levels, equal peak realized gain values, equal FTBRs, and equal 3-dB beamwidths.

As a consequence, in order to make the overall current distributions as symmetrical with respect to the z-axis as possible, more than two pairs of the driven strips were included. The outcomes from the Step III to Step VI ESAs are shown in Fig. 2(a). All the radiation patterns remained in the magnetic dipole mode oriented along $z$-axis. Moreover, the surface current distribution on the outer wall of the feedline becomes slightly more symmetrical with each increase in the number of strips. However, as more magnetic NFRP elements are added, the overall electrical size, $k a$, also increases significantly as observed from Fig. 2(b).

As a compromise to realize an antenna that would be pattern-reconfigurable over the entire azimuthal plane, be electrically small, and be as simple as possible, the Step IV design was selected. It could beam switch over three sectors with an angular interval at least as large as $120^{\circ}$ and, hence, could dynamically cover the entire azimuth plane. Note that one could select Step VI (or even a higher step) to provide a total of 5 (or more) reconfigurable states to cover the entire azimuthal plane. Such a choice would provide even lower instantaneous gain fluctuations in any specific direction, but this enhancement would be expensive. A much larger electric size and more slots and lumped elements would be necessary, leading to a corresponding increase in the design and fabrication complexities and, hence, cost. In addition, one could reduce the electrical sizes of the CLLs and, hence, the entire system by introducing lump elements into their gaps or by tailoring the gaps to act as interdigitated capacitors. These choices introduce additional complications with lumped element and fabrication tolerances. The three sector choice provided a robust means to demonstrate the efficacy of the electrically small reconfigurable design.

\section{B. Behavior of the set of electric NFRP elements}

The other main radiators of the reconfigurable Huygens source ESA are the set of three electric NFRP elements. The three sectors of the slotted-based disc each produce currents that are equivalent to a meander-line electric dipole element. These currents are illustrated in Fig. 3. The choice of a meander-line configuration for this parasitic element achieves its miniaturization [39]. With the connecting PIN diode in its ON state, the current can pass between the two sectors, effectively doubling the overall length of the main meander-line element and allowing it to go into resonance. The currents on the remaining sector act as another electric dipole. It helps provide the proper phase difference so that the two-element array, being centered along the desired beam direction, will create the appropriate electric dipole response which, when combined with the magnetic NFRP element generated magnetic dipole, will produce the desired Huygens source behavior.

To further explain this "two-element dipole array" operational mechanism, the induced surface current distributions on the three electric NFRP elements over a half-period of the source excitation are presented in Fig. 3 when the diodes are selected to form the cardioid pattern with its maximum along the $+\mathrm{x}$-axis. The source phase $\psi$ was varied from 0 to $\pi$ in steps of $\pi / 12$; only the results at multiples of $\pi / 6$ are presented. This scan allowed a detailed study of the current magnitude and phase behaviors as the source excites the structure. It is observed that almost equal current amplitudes are present on the longer two-sector and the shorter one-sector parasitic elements. Nevertheless, even though they are the same distance away from the driven element, their phase responses are quite different. Specifically, the current direction changes from clockwise to counter-clockwise at a fixed phase angle, $\psi_{2}$, within the range $[\pi / 2,7 \pi / 12]$ for the longer meander-line, while it changes in the reverse manner on the shorter meander-line at a

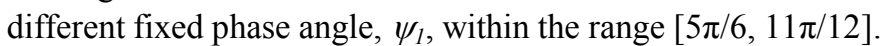
Thus, it is easily calculated that the minimum and maximum phase differences that can exist between the two electric NFRP element sectors (i.e., between the longer and the shorter meander-lines) are within the range $[(\pi / 2-11 \pi / 12),(7 \pi / 12$ $5 \pi / 6)]=[-5 \pi / 12,-\pi / 4]=\left[-75^{\circ},-45^{\circ}\right]$.

The array factor for a two-element dipole array with both dipoles oriented in the same direction and with the same 
excitation magnitude should theoretically be $f_{\text {array }}=\left|1+\mathrm{e}^{\mathrm{j} u}\right|$, where $u=k d \cos \varphi+\Delta \psi, d$ being the distance between the two dipoles (i.e., the distance between the centers of the two electric NFRP element sectors, which is $35 \mathrm{~mm}\left(\sim 0.184 \lambda_{0}\right)$ in our design); $\Delta \psi$ being the phase difference between them; and $\varphi$ being the main-beam angle measured from $+x$-axis [40]. Therefore, the two sectors are in phase and will produce an end-fire response [41] when $u$ is equal to zero, i.e., when the main-beam direction is $\varphi= \pm \arccos [-\Delta \psi /(k d)]$. For the allowed phase differences, this means that $\varphi$ will be within the interval $\left[-47.2^{\circ}, 47.2^{\circ}\right]$. The distance $d$ was optimized to ensure that the Huygens antenna's main-beam direction was at the center of this interval, i.e., along the $+x$-axis. On the other hand, in the opposite direction, $u=\pi$ and a null is formed. Thus, the cardioid pattern is obtained from this effective two-element array.

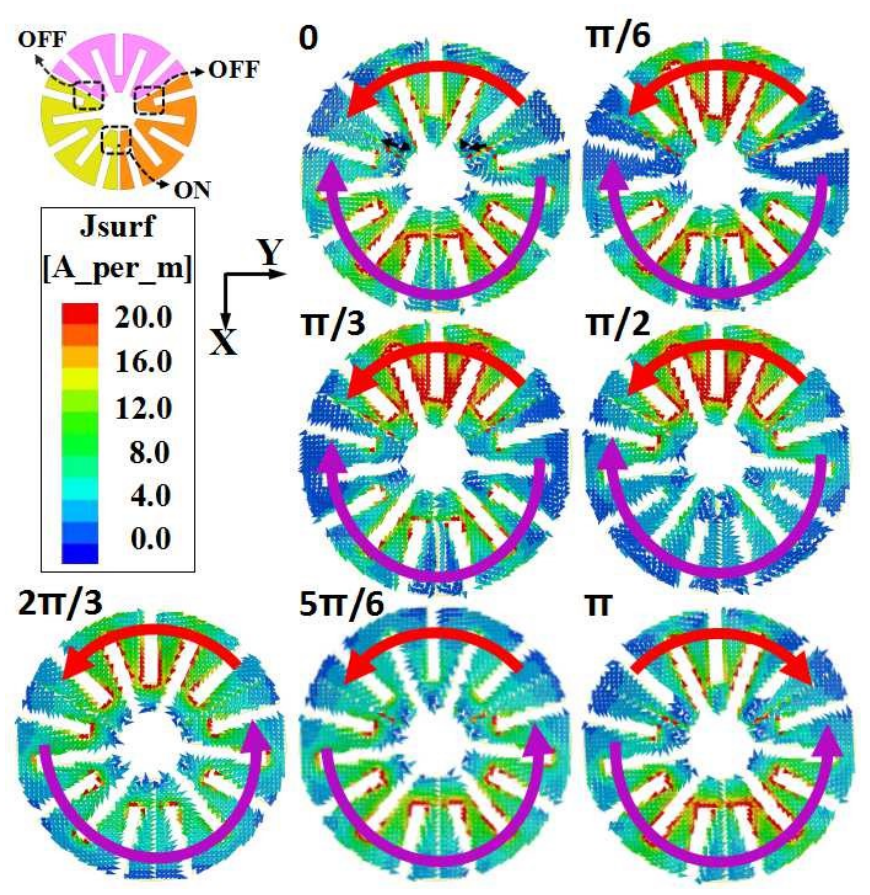

Fig. 3 Surface current distributions on the upper face of the disk supporting the reconfigurable electric element of the pattern-reconfigurable Huygens antenna in State A are shown over a half-period of the source excitation phase $\psi$. While $\pi / 12$ phase steps were examined in detail, only the results for multiples of $\pi / 6$ are shown. Note that $0^{\circ}$ is down, along the $+x$-axis. The current behavior on the shorter strip is labeled as a red arrow and that on the longer strip is labeled as the purple arrow

To further confirm this behavior, the radiation performance of the optimized antenna was studied parametrically using three unequal sectors. Fig. 4 gives the 2D directivity patterns in the $z x$-plane when the angles between the two OFF diodes are changed in the angular range: $\left[100^{\circ}, 140^{\circ}\right]$, while the other parameters remain unchanged. It was found that the main-beam angle $\varphi$ shifted from positive $\left(14^{\circ}\right)$ to negative values $\left(-19^{\circ}\right)$ as the OFF-state difference angle was increased. Clearly, the desired behavior is obtained with the $120^{\circ}$ choice. This process of changing the angle between the two NFRP elements is equivalent to adjusting the magnitude and phase of the currents induced on them, resulting in a shift of the main-beam direction $\varphi$ within a certain angular range.

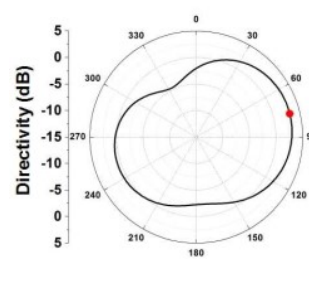

(a)

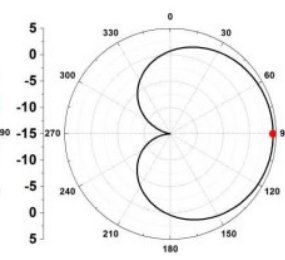

(b)

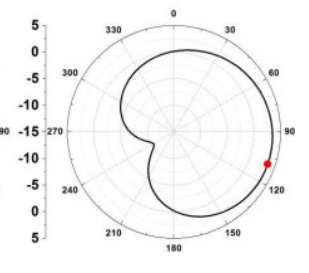

(c)
Fig. 4 2D directivity patterns in the $z x$-plane when the angle between the two OFF diodes is varied: (a) $100^{\circ}$, (b) $120^{\circ}$, and (c) $140^{\circ}$, while the other design parameters remain fixed. The main-beam direction is indicated as a red point in each sub-figure.

In addition, it should be noted that the associated end-fire array design principles could be extended to more equal sectors, realizing lower gain fluctuations. Nevertheless, it is again clear that this would add complexity and costs since it would entail an even more delicate balance between increased numbers of effective electric dipoles and would require a more careful arrangement and suitable control of the PIN diodes to achieve the required phase differences between them [41]. The three-sector design suitably demonstrates the desired performance characteristics.

\section{Effect of the gap between the substrates}

Table II. The EfFect of the Distance Between the Two Substrates, h1, on The ANTEnNa Performance Characteristics

\begin{tabular}{|c|c|c|c|c|c|}
\hline $\begin{array}{c}\mathrm{h} 1 \\
(\mathrm{~mm})\end{array}$ & 4 & 6 & 8 & 10 & 12 \\
\hline $\begin{array}{c}\text { Res. } \\
\text { frequency } \\
(\mathrm{GHz})\end{array}$ & 1.628 & 1.605 & 1.577 & 1.561 & 1.553 \\
\hline $\begin{array}{c}\left|\mathrm{S}_{11}\right| \min \\
(\mathrm{dB})\end{array}$ & -26.53 & -22.11 & -20.92 & -33.51 & -20.43 \\
\hline $\begin{array}{c}\text { Peak realized } \\
\text { gain (dBi) }\end{array}$ & 2.45 & 3.54 & 3.99 & 4.09 & 3.87 \\
\hline $\begin{array}{c}\text { FTBR } \\
(\mathrm{dB})\end{array}$ & 3.795 & 7.833 & 20.126 & 13.84 & 9.549 \\
\hline $\begin{array}{c}\text { Radiation } \\
\text { efficiency (\%) }\end{array}$ & 92.2 & 92.69 & 91.04 & 91.83 & 91.9 \\
\hline
\end{tabular}

The effect of the gap between the two substrates, i.e., the distance $\mathrm{h} 1$, on the antenna performance characteristics was also investigated. The associated simulation results are listed in Table II. They demonstrate that with a decrease of h1, the electric NFRP elements are moved closer to the driven element. Their increased proximity causes not only an increase of the capacitive coupling level between the electric NFRP and driven elements, but it also adjusts the relative phases between the electric and magnetic NFRP elements. As indicated by the predicted outcomes, this combination of coupling levels and phase responses achieves the optimal Huygens source radiation behavior [33-36] when $\mathrm{h} 1=8 \mathrm{~mm}$. It is noted that when h1 is smaller than $8 \mathrm{~mm}$, there is an over-coupling of the electric 
NFRP and driven elements which leads to unbalanced induced current magnitudes between the electric and magnetic NFRP elements. This effect seriously degrades the Huygens source radiation performance, especially the resulting FTBR value.

D. E-field behavior in the near field of the pattern-reconfigurable Huygens ESA

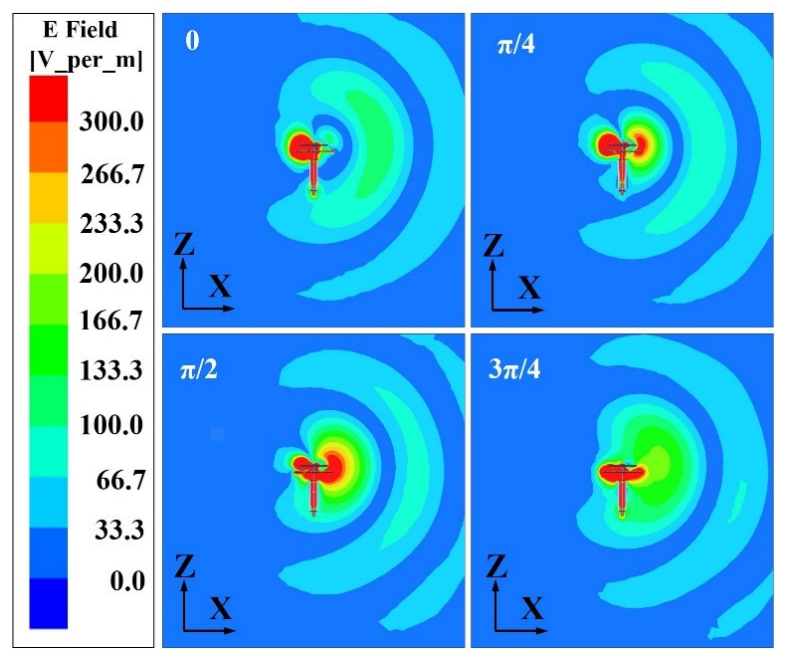

Fig. 5 The simulated near-field $E$-field distributions in the zx plane of the pattern-reconfigurable Huygens ESA in State A for different excitation phases over a half period.

From the two previous sub-sections, one can conclude that the driven element has two functions. First, the magnetic element is coupled to it to produce the appropriate magnetic dipole response. Second, the reconfigurable electric element is capacitively coupled to it in a manner to produce a two-element end-fire array response. To further understand the radiation mechanism of the entire pattern-reconfigurable Huygens ESA, the $E$-field distributions in its near field for several excitation phases are plotted in Fig. 5. One can see that the sum of the patterns from the one magnetic dipole NFRP element and the two electric dipole NFRP elements generate the desired Huygens source behavior [33-36]. However, because the phase responses of the electric and magnetic NFRP elements are not precisely in phase, especially at $3 \pi / 4$, the resulting $3-\mathrm{dB}$ beamwidth in the vertical plane is $\sim 160^{\circ}$, much wider than the analytical Huygens source value, i.e., $\sim 131^{\circ}$ in $[33,34,36]$. Finally, it is noted that because of the presence of the sleeve balun in the experiments to eliminate any concerns of stray currents on the outer wall of the coax-feed line, almost no electromagnetic energy leakage occurred as was expected from previous measurement campaigns $[42,43]$.

\section{Simulated Antenna Performance AND EXPERIMENTAL VALIDATION}

A prototype of the simulated reconfigurable, Huygens source ESA was fabricated, assembled, and measured. Photographs of the fabricated prototype are given in Fig. 6. A sleeve balun was employed in the measurements to choke-off the leakage currents on the outer wall of the measurement cable.
As the antenna under test (AUT), it thus maintained the predicted impedance matching and the predicted the near- and far-field radiation pattern characteristics $[42,43]$.

A GWinstek GPC-3060D DC voltage-stabilized power supply is applied to feed $\sim 1.4 \mathrm{~V}$ voltages to the diodes connecting the antenna's electric NFRP elements. As shown in Figs. 6(b) and 6(c) with their noted colors, different pairs of DC lines were used to switch the diodes between their ON and OFF states. Recall that the three diodes are denoted by their placement in the clockwise direction shown in Fig. 1(e). State A employs the yellow ("+" pole) and white ("-" pole) DC lines; State B employs the white ("+" pole) and black ("“-" pole) DC lines; and State C employs the black ("+" pole) and yellow ("“-" pole) DC lines.

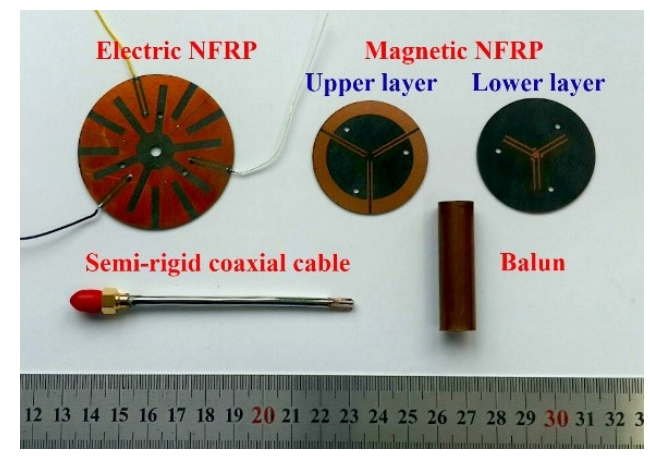

(a)

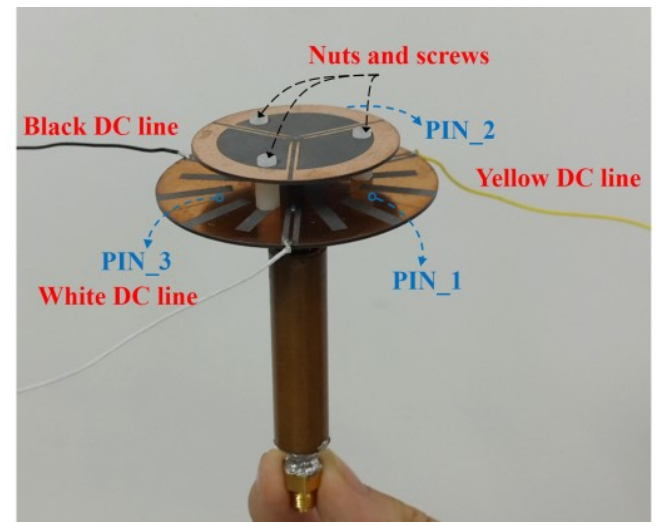

(b)

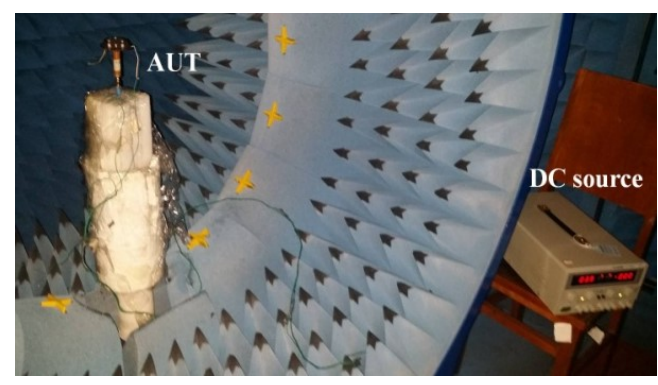

(c)

Fig. 6 The fabricated reconfigurable ESA prototype in the measurement chamber with the sleeve balun installed. (a) The antenna elements before assembly, (b) side view of the prototype antenna after assembly, and (c) the prototype antenna as the AUT in the chamber. 
The values of the magnitude of the reflection coefficient, $\left|\mathrm{S}_{11}\right|$, were measured as a function of the source frequency for all three states using an Agilent E8361A PNA Vector Network Analyzer (VNA). The measured results, together with their simulated values, are given in Fig. 7. The measured results indicate that the antenna is nearly completely impedance matched at $1.5622,1.5613$, and $1.5677 \mathrm{GHz}$ for States A, B, and C, respectively, where $\left|\mathrm{S}_{11}\right|_{\min }=-28.88,-29.99$, and $-24.84 \mathrm{~dB}$. The corresponding $-10 \mathrm{~dB}$ bandwidths are, respectively, 42.7 , 37.4 , and $35.5 \mathrm{MHz}$, i.e., a fractional bandwidth equal to $2.73 \%$, $2.40 \%$, and $2.26 \%$. The antenna is thus electrically small with $k a \sim 0.92$ and low profile with $h \sim 0.05 \lambda_{0}$, where $\lambda_{0}$ is the free space wavelength for all three resonance frequencies.

We take the average of the three measured states: 1.564 $\mathrm{GHz}$, as their representative frequency. In comparison, the simulated $\left|\mathrm{S}_{11}\right|_{\min }$ values (all below $-20 \mathrm{~dB}$ ) are located at 1.577 $\mathrm{GHz}$ with a $43.1 \mathrm{MHz}$ bandwidth, i.e., a $2.73 \%$ fractional bandwidth, in every state. Thus, the experimental results exhibit only about a $0.015 \mathrm{GHz}(15 \mathrm{MHz})$ red shift from the simulated ones, i.e., they are in good agreement ( $<1 \%$ difference).

Additionally, as is also shown in Fig. 7 in the sub-plots, the simulated radiation main-beam direction at the resonance frequency $1.577 \mathrm{GHz}$ in each of the three states is, respectively, in the $0 \mathrm{deg}, 120 \mathrm{deg}$ and 240 degree directions in the azimuth plane (i.e., in xy plane). Furthermore, the maximum lies in the xy plane in those directions. For all the three states, the simulated peak realized gain $\left(R_{\max }\right)$, front-to-back ratio (FTBR), and radiation efficiency (RE) values are $\sim 3.98 \mathrm{dBi}$, $\sim 20.13 \mathrm{~dB}$, and $\sim 91.05 \%$, respectively. It is important to note that our simulation studies showed that when the antenna has the same feed-line length $(70 \mathrm{~mm})$ but without the balun, the $\mathrm{RG}_{\max }$, FTBR, and RE values are $\sim 3.96 \mathrm{dBi}, \sim 13.64 \mathrm{~dB}$, and $\sim 90.58 \%$, respectively. Consequently, the measured results further confirm that the contribution of the balun is simply to maintain the performance characteristics of the prototype ESA during the various measurement procedures.

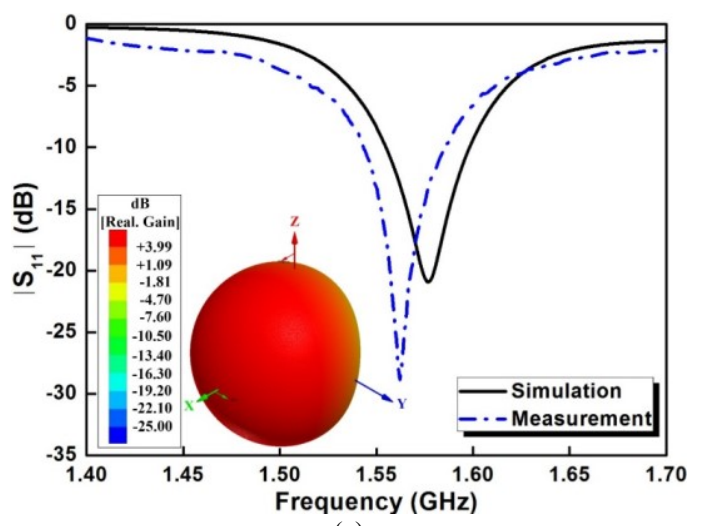

(a)

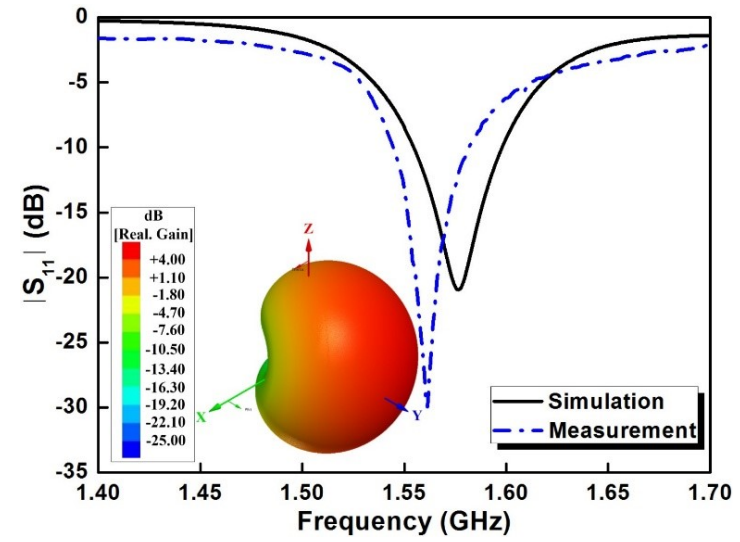

(b)

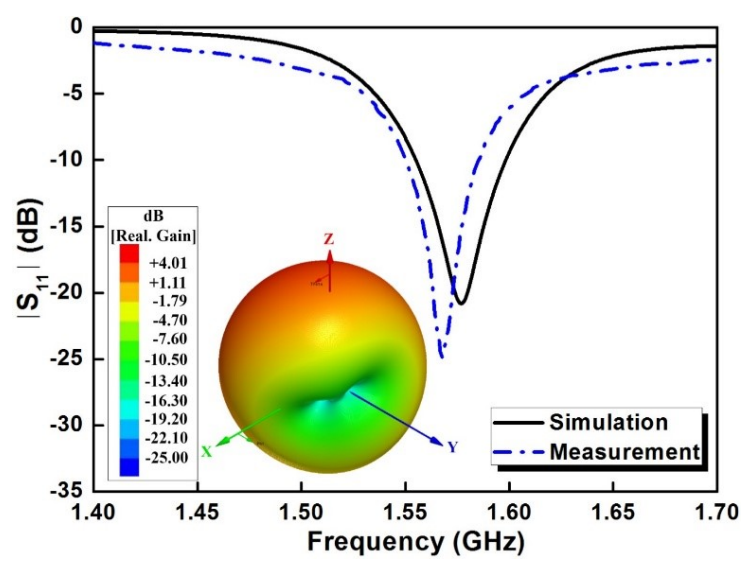

(c)

Fig. 7 The measured and simulated values of $\left|\mathrm{S}_{11}\right|$ (reflection coefficient values) as functions of the source frequency for the reconfigurable Huygens ESA in all three diode states, together with their corresponding simulated 3D radiation patterns at the resonance frequency $1.577 \mathrm{GHz}$. (a) State A, (b) State B, and (c) State C.

The far-field radiation performance characteristics shown in Fig. 8 were measured in an anechoic chamber at the National Key Laboratory of Science and Technology on Communications, University of Electronic Science and Technology of China (UESTC). This facility is mainly based on the Agilent N5230A PNA-L VNA and SATIMO passive measurement system $(0.8 \mathrm{GHz}-6 \mathrm{GHz})$ [44]. The prototype AUT is shown in Fig. 6(c).

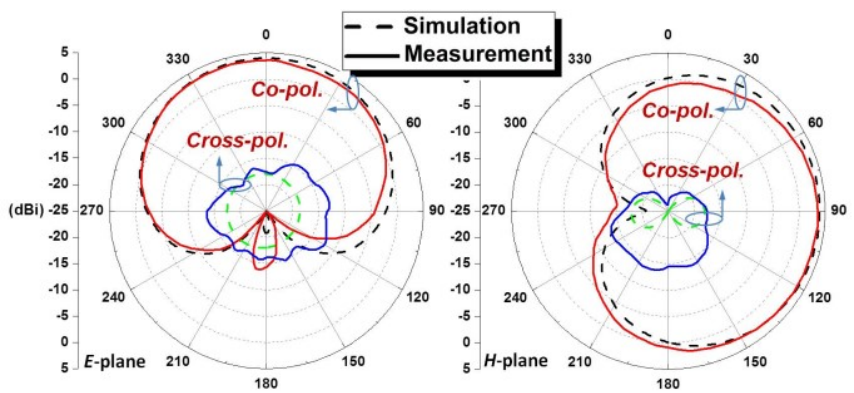

(a) 


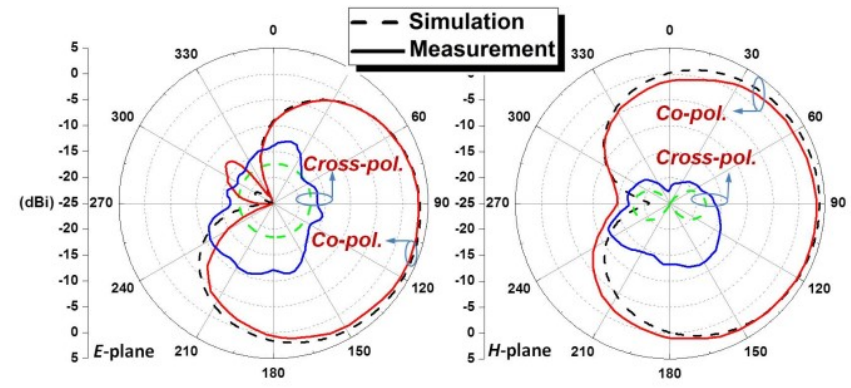

(b)

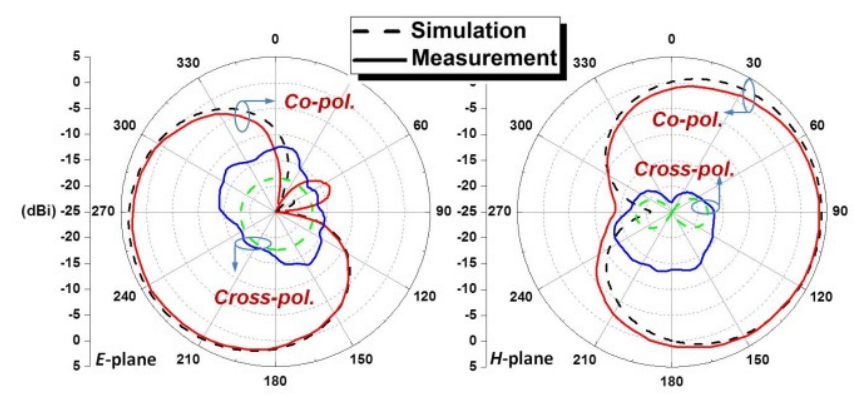

(c)

Fig. 8 Measured and simulated realized gain patterns in the $E$ (horizontal) plane and $H$ (vertical) - plane at the operational frequencies of the three states. (a) State A, (b) State B, and (c) State C.

The measured and corresponding simulated realized gain patterns are compared in Fig. 8 at the three resonance frequencies corresponding to the three diodes states. They are all in good agreement. As expected, when feeding the different PIN diodes, the main-beam direction of the antenna is changed accordingly. In particular, the radiation patterns in the three states are as follows. In State A, the measured (simulated) $3 \mathrm{~dB}$-beam coverage is from $-68^{\circ}$ to $62^{\circ}$ (from $-66^{\circ}$ to $66^{\circ}$ ) in the $E$-plane (xy plane) and from $26^{\circ}$ to $184^{\circ}$ (from $8^{\circ}$ to $170^{\circ}$ ) in the $H$-plane. In State B, the measured (simulated) $3 \mathrm{~dB}$-beam coverage is from $48^{\circ}$ to $178^{\circ}$ (from $53^{\circ}$ to $187^{\circ}$ ) in the $E$-plane and from $24^{\circ}$ to $184^{\circ}$ (from $8^{\circ}$ to $170^{\circ}$ ) in the $H$-plane. In State $\mathrm{C}$, the measured (simulated) $3 \mathrm{~dB}$-beam coverage is from $172^{\circ}$ to $304^{\circ}$ (from $173^{\circ}$ to $306^{\circ}$ ) in the $E$-plane and from $26^{\circ}$ to $184^{\circ}$ (from $8^{\circ}$ to $170^{\circ}$ ) in the $H$-plane. On the whole, with the overlap of the measured instantaneous 3dB-beamwidths in all three states, the entire azimuth plane was covered. The detailed experimental and simulation results of the reconfigurable ESA in all three states are summarized in Table III for easy comparisons in a comprehensive manner. For all the three states, the measured $\mathrm{RG}_{\max }$, FTBR, and RE values are $\sim 3.55 \mathrm{dBi}$, $17.5 \mathrm{~dB}$, and $\sim 84.9 \%$, respectively.

The experimental results witness very acceptable differences from their simulation values as is clear from Figs. 7 and 8 and from Table III. The various differences are mainly ascribed to the following three factors. First, because they are lumped elements, the performance characteristics of the three PIN diodes used in the experiment are not perfectly the same. Second, while it is believed to be quite minor for this antenna, the actual presence of the long DC wires was not considered in the simulations. Third, normal minor errors arising from the fabrication, assembly, installation, and experimental process are present and could not be avoided. On the whole, the measurement and simulation results agree very well.

TABLE III. THE EXPERIMENTAL AND SIMULATED RESULTS FOR THE RECONFIGURABLE HUYGENS ESA IN ALL THREE DiODE STATES (THE MEASURED RESUlTS ARE IN THE ClEAR BOXES)

\begin{tabular}{|c|c|c|c|}
\hline State & $\mathrm{A}$ & $\mathrm{B}$ & $\mathrm{C}$ \\
\hline \multirow{2}{*}{$\begin{array}{c}\text { Res. frequency } \\
(\mathrm{GHz})\end{array}$} & 1.577 & 1.577 & 1.577 \\
\cline { 2 - 4 }$\left|\mathrm{S}_{11}\right|$ min $(\mathrm{dB})$ & 1.562 & 1.561 & 1.568 \\
\hline \multirow{2}{*}{ Bandwidth (\%) } & -20.92 & -20.94 & -20.83 \\
\cline { 2 - 4 } & -28.88 & -29.99 & -24.84 \\
\cline { 2 - 4 } & 2.73 & 2.73 & 2.73 \\
\hline $\begin{array}{c}\text { Main-beam direction in } \\
\text { horizontal plane (degree) }\end{array}$ & 2.73 & 2.40 & 2.26 \\
\cline { 2 - 4 } & -2 & 120 & 240 \\
\hline \multirow{2}{*}{ Peak realized gain (dBi) } & 3.9878 & 3.9925 & 4.0015 \\
\cline { 2 - 4 } & 3.59 & 3.58 & 3.52 \\
\hline \multirow{2}{*}{ Radiation efficiency (\%) } & 91.04 & 91.07 & 91.06 \\
\cline { 2 - 4 } & 85.07 & 84.79 & 84.92 \\
\hline \multirow{2}{*}{ FTBR (dB) } & 20.126 & 20.1713 & 20.2444 \\
\cline { 2 - 4 } & 17.94 & 18.41 & 17.36 \\
\hline $\begin{array}{c}\text { 3-dB beam coverage in } \\
\text { horizontal -plane (degree) }\end{array}$ & $-66 \sim 66$ & $53 \sim 187$ & $173 \sim 306$ \\
\cline { 2 - 4 } & $-68 \sim 62$ & $48 \sim 178$ & $172 \sim 304$ \\
\hline \multirow{2}{*}{$\begin{array}{c}\text { 3-dB beamwidth in } \\
\text { horizontal -plane (degree) }\end{array}$} & 132 & 134 & 133 \\
\cline { 2 - 4 } & 130 & 130 & 132 \\
\hline $\begin{array}{c}\text { 3-dB beam coverage in } \\
\text { vertical -plane (degree) }\end{array}$ & $8 \sim 170$ & $8 \sim 170$ & $8 \sim 170$ \\
\cline { 2 - 4 } & $26 \sim 184$ & $24 \sim 184$ & $26 \sim 184$ \\
\hline $\begin{array}{c}\text { 3-dB beamwidth in } \\
\text { vertical -plane (degree) }\end{array}$ & 162 & 162 & 162 \\
\cline { 2 - 4 } & 158 & 160 & 158 \\
\hline
\end{tabular}

TABLE IV. COMPARISON OF THE PATTERN-RECONFIGURABLE HUYGENS ANTENNA WITH RELATED ANTENNAS REPORTED IN THE LITERATURE

\begin{tabular}{|c|c|c|c|c|c|c|}
\hline Category & ka & $\begin{array}{c}\text { FBW } \\
(\%)\end{array}$ & $\begin{array}{c}\text { Peak } \\
\text { gain } \\
(\mathrm{dBi})\end{array}$ & $\begin{array}{c}\text { Switch } \\
\text { No. }\end{array}$ & $\begin{array}{c}\text { Covering } \\
\text { space }\end{array}$ & $\begin{array}{c}\text { RE } \\
(\%)\end{array}$ \\
\hline$[12]$ & 8.38 & 20 & 4.7 & 5 & $360^{\circ}$ & 49 \\
\hline$[13]$ & 3.43 & 4 & 6.5 & 12 & - & - \\
\hline$[14]$ & 4.37 & 3.64 & 7.27 & 8 & $360^{\circ}$ & 86.1 \\
\hline$[15]$ & 1.84 & $\sim 2.7$ & 1.23 & 8 & $360^{\circ}$ & - \\
\hline$[16]$ & 3.79 & $\sim 1$ & - & 4 & $140^{\circ}$ & - \\
\hline$[17]$ & 4.51 & 40 & 5.08 & 4 & $360^{\circ}$ & 73.3 \\
\hline$[18]$ & 6.91 & 3.4 & 7.8 & 6 & $\sim 180^{\circ}$ & - \\
\hline$[19]$ & 4.62 & 6.2 & 9.94 & 12 & $90^{\circ}$ & 97 \\
\hline$[20]$ & 0.13 & 1.2 & -17.94 & 2 & $191^{\circ}$ & 0.44 \\
\hline$[23]$ & $\sim 0.6$ & 2.7 & 8.5 & 2 & $\sim 220^{\circ}$ & 89.8 \\
\hline$[24]$ & 0.98 & 2.8 & 2.21 & - & $<180^{\circ}$ & 95 \\
\hline This work & 0.92 & 2.73 & 3.59 & 3 & $360^{\circ}$ & 85.07 \\
\hline
\end{tabular}

Finally, it is noted that our innovative design is simpler and more compact than many pattern-reconfigurable antennas reported in the literature. Table IV provides a comparison listing their electrical sizes, bandwidths, end-fire peak gains, numbers of diodes employed, and reconfigurable beamwidths. Besides illustrating the advantages of its physical design, Table IV also highlights that it produces very attractive coverage of the entire azimuthal plane.

\section{CONCLUSIONS}

A simple, efficient, pattern-reconfigurable, Huygens source ESA was obtained by in series integrating three PIN diodes into 
the electric element. A prototype was fabricated and experimental confirmation of its reconfigurable performance characteristics was obtained. Very good agreement between the simulated and measured results was presented. The principles of operation of the fabricated prototype were explored in a comprehensive manner. It has many potential applications in a variety of wireless systems.

\section{REFERENCES}

[1] H. Choo, R. L. Rogers, and H. Ling "Design of electrically small wire antennas using a Pareto genetic algorithm," IEEE Trans. Antennas Propag., vol. 53, no. 3, pp. 1038-1046, Mar. 2005.

[2] J. L. Volakis, and K. Sertel, "Narrowband and wideband metamaterial antennas based on degenerate band edge and magnetic photonic crystals," Proc. IEEE, vol. 99, no. 10, pp. 1732-1745, Oct. 2011.

[3] Y. Dong and T. Itoh, "Metamaterial-based antennas," Proc. IEEE, vol. 100, no. 7, pp. 2271-2285, Jul. 2012.

[4] J. Oh, and K. Sarabandi, "Low profile vertically polarized Omnidirectional wideband antenna with capacitively coupled parasitic element," IEEE Trans. Antennas Propag., vol.62, no. 2, pp. 977- 982, Feb. 2014.

[5] M. M. H. Armanious, S. V. B. Olaya, J. S. Tyo, M. C. Skipper, M. D. Abdalla, L. L. Altgilbers, and A. Bryan, "An electrically small conical folded dipole antenna for use as a compact, self-resonant mesoband high-power microwave source," IEEE Trans. Antennas Propag., vol.62, no. 12, pp. 5960- 5967, Dec. 2014.

[6] J. Zhang, and Y. Long, "A novel metal-mountable electrically small antenna for RFID tag applications with practical guidelines for the antenna design," IEEE Trans. Antennas Propag., vol.62, no. 11, pp. 5820- 5829, Nov. 2014.

[7] S. R. Best, "Electrically small resonant planar antennas: Optimizing the quality factor and bandwidth," IEEE Antennas Propag. Mag., vol. 57, no. 3, pp. 38-47, Jun. 2015.

[8] M. Hosseini, D. M. Klymyshyn, G. Wells, and X. Liu, "Short and open circuited EBG resonator antennas: miniaturization with a shorting plate and dielectric loading," IEEE Trans. Antennas Propag., vol. 63, no. 10, pp. 4305-4314, Oct. 2015.

[9] R. W. Ziolkowski, P. Jin, and C.-C. Lin, "Metamaterial-inspired engineering of antennas," Proc. IEEE, vol. 99, no. 10, pp. 1720-1731, Oct. 2011.

[10] A. Petosa, "An overview of tuning techniques for frequency-agile antennas," IEEE Antennas Propag. Mag., vol. 54, no.5, pp. 271-296, Oct. 2012.

[11] C. G. Christodoulou, Y. Tawk, S. A. Lane, and S. R. Erwin, "Reconfigurable antennas for wireless and space applications," Proc. IEEE, vol. 100, no. 7, pp. 2250-2261, Jul. 2012.

[12] J.-S. Row and C.-W. Tsai "Pattern reconfigurable antenna array with circular polarization," IEEE Trans. Antennas Propag., vol. 63, no. 1, pp. 24-32, Jan. 2015.

[13] Z. Li, E. Ahmed, A. M. Eltawil, and B. A. Cetiner "A Beam-steering reconfigurable antenna for WLAN applications," IEEE Trans. Antennas Propag., vol. 64, no. 4, pp. 1525-1530, Apr. 2016.

[14] L. Zhong, J.-S. Hong, and H.-C. Zhou, "A novel pattern-reconfigurable cylindrical dielectric resonator antenna with enhanced gain," IEEE Antennas Wirel. Propag. Lett., vol. 15, pp. 1253-1256, 2016.

[15] X. Cheng, Y. Yao, S.-W. Qu, Y. Wu, J. Yu, and X. Chen, "Circular beam-reconfigurable antenna base on graphene-metal hybrid," Electron. Lett., vol. 52, no. 7, pp. 494- 496, Apr. 2016.

[16] Y. Bai, S. Xiao, M.-C. Tang, Z.-F. Ding, B.-Z Wang, "Wide-angle scanning phased array with pattern reconfigurable elements," IEEE Trans. Antennas Propag., vol. 59, no. 11, pp. 4071 - 4076, Nov. 2011.

[17] T. Zhang, S.-Y. Yao, and Y. Wang, "Design of radiation-pattern-reconfigurable antenna with four beams," IEEE Antennas Wirel. Propag. Lett., vol. 14, pp. 183-186, 2015.

[18] T. Sabapathy, M. Jusoh, R. B. Ahmad, M.R. Kamarudin, and P. J. Soh, "A ground-plane-truncated, broadly steerable Yagi-Uda patch array antenna," IEEE Antennas Wirel. Propag. Lett., vol. 15, pp. 1069-1072, 2016.

[19] N. Ramli, M. T. Ali, M. T. Islam, A. L. Yusof, and S. Muhamud-Kayat,
"Aperture-coupled frequency and patterns reconfigurable microstrip stacked array antennas," IEEE Trans. Antennas Propag., vol. 63, no. 3, pp. 1067 - 1074, Mar. 2015.

[20] V. T. Nguyen, and C. W. Jung, "Radiation-pattern reconfigurable antenna for medical implants in MedRadio band," IEEE Antennas Wirel. Propag. Lett., vol. 15, pp. 106-109, 2016.

[21] R. F. Harrington, "On the gain and beamwidth of directional antennas," IEEE Trans. Antennas Propag., vol. 6, no. 3, pp. 219-225, Jul. 1958.

[22] C. A. Balanis, Antenna Theory: Analysis and Design, 3rd edition, New York: Wiley Interscience, 2005.

[23] S. Lim, and H. Ling, "Design of electrically small, pattern reconfigurable Yagi antenna," Electron. Lett., vol. 43, no. 24, pp. 1326-1327, Nov. 2007.

[24] M.-C. Tang, T. Shi, and R. W. Ziolkowski, "Electrically small uniplanar antenna with pattern-agile performance," Electron. Lett., vol. 51, no. 16, pp. 1228 - 1229, Aug. 2015.

[25] S. Lim and H. Ling, "Design of electrically small Yagi antenna," Electron. Lett., vol. 43, no. 5, pp. 3-4, Mar. 2007.

[26] R. Baggen, M. Martínez-Vázquez, J. Leiss, S. Holzwarth, L. Salghetti Drioli and P. de Maagt, "Low profile GALILEO antenna using EBG technology," IEEE Trans. Antennas Propag., vol. 56, pp. 667-674, Mar. 2008.

[27] F. Yang and Y. Rahmat-Samii, Electromagnetic Band Gap Structures in Antenna Engineering, Cambridge, UK: Cambridge University Press, 2009.

[28] R. L. Li, B. Pan, A. N. Traille, J. Papapolymerou, J. Laskar, and M. M. Tentzeris, "Development of a cavity-backed broadband circularly polarized slot/strip loop antenna with a simple feeding structure," IEEE Trans. Antennas Propag., vol. 56, no. 2, pp. 312-318, Feb. 2008.

[29] P. Alitalo, A. O. Karilainen, T. Niemi, C. R. Simovski, and S. A. Tretyakov, "Design and realisation of an electrically small Huygens source for circular polarization," IET Microwaves, Antennas \& Propagation, vol. 5, no. 7, pp. 783-789, 2011.

[30] K.-M. Luk, and B. Wu, "The magnetoelectric dipole: A wideband antenna for base stations in mobile communications," Proc. IEEE, vol. 100, no. 7, pp. 2297-2307, Jul. 2012.

[31] C. Morlaas, B. Souny, and A. Chabory, "Helical-ring antenna for hemispherical radiation in circular polarization," IEEE Trans. Antennas Propag., vol. 63, no. 11, pp. 4693-4701, Nov. 2015.

[32] H.-J. Seo, and A. A. Kishk, "Wideband magnetic-electric antenna with linear single or dual polarization," Progress In Electromagnetics Research (PIER), vol. 155, pp. 53-61, Mar. 2016.

[33] P. Jin, and R. W. Ziolkowski, "Metamaterial-inspired, electrically small, Huygens sources," IEEE Antennas Wireless Propag. Lett., vol. 9, pp. 501-505, May 2010.

[34] M.-C. Tang, H. Wang, and R. W. Ziolkowski, "Design and testing of simple, electrically small, low-profile, Huygens source antennas with broadside radiation performance," IEEE Trans. Antennas Propag., vol. 64, no. 11, pp. 4607-4617, Nov. 2016.

[35] R. W. Ziolkowski, "Low profile, broadside radiating, electrically small Huygens source antennas," IEEE Access, vol. 3, pp. 2644-2651, Dec. 2015.

[36] M.-C. Tang, T. Shi, and R. W. Ziolkowski, "Electrically small, broadside radiating Huygens source antenna augmented with internal non-Foster elements to increase its bandwidth," IEEE Antennas Wirel. Propag. Lett., early access, DOI:10.1109/LAWP.2016.2600525, Jul. 22, 2016.

[37] ANSYS/ANSOFT. (2012). High Frequency Structure Simulator (HFSS), ver. 13.0 [Online]. Available: www.ansoft.com.

[38] http://cdn.macom.com/datasheets/MA4GP907.pdf

[39] M.-C. Tang, and R. W. Ziolkowski, "Efficient, high directivity, large front-to-back-ratio, electrically small, near-field-resonant-parasitic antenna," IEEE Access, vol. 1, no. 1, pp. 16 - 28, May 2013.

[40] T. A. Milligan, Modern Antenna Design, 2nd edition, New Jersey: John Wiley \& Sons, 2005.

[41] E. E. Altshuler, T. H. O'Donnell, A. D. Yaghjian, and S. R. Best, “A monopole superdirective array," IEEE Trans. Antennas Propag., vol. 53, no. 8, pp. 2653-2661, Aug. 2005.

[42] C. Icheln, J. Krogerus, and P.Vainikainen, "Use of balun chokes in small-antenna radiation measurements," IEEE Trans. Antennas Propag., vol.53, no. 2, pp.498-506, Apr. 2004

[43] M.-C. Tang, and R. W. Ziolkowski, "A study of low-profile, broadside radiation, efficient, electrically small antennas based on complementary 
split ring resonators," IEEE Trans. Antennas Propag., vol. 61, no. 9, pp. 4419 - 4430, Sep. 2013.

[44] StarLab Version D User Guide 1.0, Reference: TD. 224.1.08. SATF. A, SATIMO Corporations 2008.

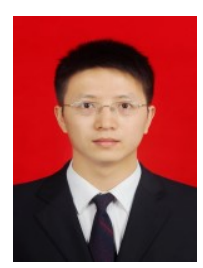

Ming-Chun Tang (S'12-M'13-SM'16) received the B. S. degree in physics from the Neijiang Normal University, Neijiang, China, in 2005 and the Ph. D. degree in radio physics from the University of Electronic Science and Technology of China (UESTC), in 2013. From August 2011 to August 2012, he was also with the Department of Electrical and Computer Engineering, The University of Arizona, Tucson, AZ, USA, as a Visiting Scholar. He is currently an Assistant Professor in the College of Communication Engineering, Chongqing University, China. His research interests include electrically small antennas, RF circuits, metamaterial designs and their applications.

Prof. Tang was a recipient of the Best Student Paper Award in the 2010 International Symposium on Signals, Systems and Electronics (ISSSE2010) held in Nanjing, China. He is serving as a reviewer for IEEE/IET journals including the IEEE Transactions on Microwave Theory and Techniques, IEEE Transactions on Antennas and Propagation, IEEE Antennas and Wireless Propagation Letters, IEEE Antennas and Propagation Magazine, IEEE Access, IET Microwaves, Antennas \& Propagation, and Electronics Letters.

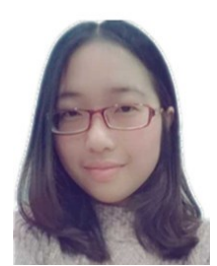

Boya Zhou (S'16) received the B.S. degree from the College of Communication Engineering, Chongqing University, China, in 2015. She is currently pursuing the M.S. degree in electronic science and technology in the College of Communication Engineering, Chongqing University, China.

Her current research interests include flexible antennas, electrically small antennas, and their applications.

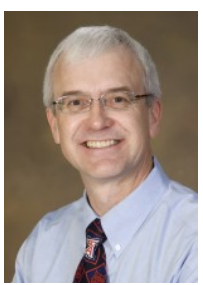

Richard W. Ziolkowski (M'87-SM'91-F'94) received the B.Sc. (magna cum laude) degree (Hons.) in physics from Brown University, Providence, RI, USA, in 1974; the M.S. and Ph.D. degrees in physics from the University of Illinois at Urbana-Champaign, Urbana, IL, USA, in 1975 and 1980, respectively; and the Honorary Doctorate degree from the Technical University of Denmark, Kongens Lyngby, Denmark in 2012.

He is currently a Distinguished Professor with the University of Technology Sydney, Global Big Data Technologies Centre, Ultimo NSW, Australia. He is also a Litton Industries John M. Leonis Distinguished Professor with the Department of Electrical and Computer Engineering and a Professor with the College of Optical Sciences at The University of Arizona. He was the Computational Electronics and Electromagnetics Thrust Area Leader with the Lawrence Livermore National Laboratory, Engineering Research Division, before joining The University of Arizona, Tucson, AZ, USA, in 1990. He was the Australian DSTO Fulbright Distinguished Chair in Advanced Science and Technology from 2014-2015. He was a 2014 Thomas-Reuters Highly Cited Researcher. His current research interests include the application of new mathematical and numerical methods to linear and nonlinear problems dealing with the interaction of electromagnetic and acoustic waves with complex linear and nonlinear media, as well as metamaterials, metamaterial-inspired structures, and applications-specific configurations.

Prof. Ziolkowski is a Fellow of the Optical Society of America (OSA, 2006), and of the American Physical Society (APS, 2016). He served as the President of the IEEE Antennas and Propagation Society in 2005. He is also actively involved with the URSI, OSA and SPIE professional societies. 